\title{
On the path toward organic spintronics
}

\author{
Jagadeesh S. Moodera, Bert Koopmans, and Peter M. Oppeneer, \\ Guest Editors
}

\begin{abstract}
Organic materials provide a unique platform for exploiting the spin of the electron-a field dubbed organic spintronics. Originally, this was mostly motivated by the notion that because of weak spin-orbit coupling, due to the small mass elements in organics and small hyperfine field coupling, organic matter typically displays a very long electron spin coherence time. More recently, however, it was found that organics provide a special class of spintronic materials for many other reasons - several of which are discussed throughout this issue. Over the past decade, there has been a growing interest in utilizing the molecular spin state as a quantum of information, aiming to develop multifunctional molecular spintronics for memory, sensing, and logic applications. The aim of this issue is to stimulate the interest of researchers by bringing to their attention the vast possibilities not only for unexpected science but also for the enormous potential for developing new functionalities and applications. The six articles in this issue deal with some of the breakthrough work that has been ongoing in this field in recent years.
\end{abstract}

\section{Introduction}

Organic molecules are inexpensive multifunctional materials that hold profound promise for realizing the goal of sustainable development. Organic molecules have almost unlimited possibilities due to multifarious options of chemical synthesis of tailor-made molecules, in combination with their mechanical flexibility and ease of large-scale development. Most molecules also respond to external stimuli such as electric and magnetic fields, light, and pressure, giving them versatility for multiple functionalities. In particular, organic materials provide a unique platform for exploiting the spin of the electron that can lead to organic spintronics. The main motivation for this directive stems from the weak coupling between the spin and orbital momentum of the electron since, typically, organics are composed of light elements. In addition, the coupling between the electron spin and nuclear magnetic moments is very small in organic matter, and the orientation of the electron spin is typically preserved for a very long time. Hence, the spin information can be maintained and transported over microscopic distances, a feature that has stimulated intensive research activities. Much of the recent research is not limited to just the breakthroughs described in this issue, but rather, these should be considered the tip of the iceberg.

\section{Molecular devices}

Besides generic interest in exploring and exploiting the spin degree of freedom in organic devices, research activities match the ongoing drive toward truly single molecular devices. The use of a single molecule as a basic functional unit to reduce device dimensions has been the goal and challenge for decades. Why single molecules? Because molecules are well defined (e.g., they always have the same number of atoms, atomic structure, and electronic structure), and they form the smallest possible electronic units that can be controlled with atomic precision. Importantly, it is possible to design their properties in limitless ways that can be precisely tuned by attaching specific functional groups. Four decades ago, for example, Aviram and Ratner envisaged a molecular rectifier based on a single molecule with donor and acceptor groups separated by a barrier. ${ }^{1}$ In addition to the charge, if one now uses the electron spin degree of freedom and hence incorporate spintronic functions within a single molecular building block, this would be the ultimate versatility for molecular spintronics.

Success in achieving spintronic devices based on singleorganic molecules relies on the control of interactions at the organic molecule-metal interfaces. While single molecules are paramagnetic, and hence the spin function cannot be employed, metal-organic molecules adsorbed on a ferromagnetic (FM) 
surface were discovered to exhibit a stable molecular spin well above room temperature. ${ }^{2}$ This property opens a route to designing spintronic devices that operate at room temperature. The crucial stabilization of the molecular spin occurs through magnetic exchange coupling at the molecule-surface spin-interface (leading to an effective electrode, which later is termed as spinterface). The adsorption of organic molecules on a FM surface to form a molecule-FM interface is, however, a complex phenomenon.

An example of such intricate magnetic coupling occurring at the molecule-surface interface is shown in Figure 1. Here, the magnetic interaction of an iron-phthalocyanine molecule adsorbed onto a cobalt substrate, computed with density functional theory (DFT), is illustrated. The magnetization densities on the $\mathrm{Fe}$ and $\mathrm{Co}$ atoms couple ferromagnetically, as signified by the orange magnetization density, but the magnetization density induced on carbon atoms couples antiferromagnetically to the Co magnetization (shown by the light-blue isosurfaces). ${ }^{3}$ This exchange interaction arising between an organic molecule and a surface determines the electronic and magnetic structure of the entire system, and thus its understanding is crucial, complex, and limitless. The binding of the molecule to the surface through charge transfer between the molecule and surface (present in both physisorption and chemisorption processes) dictates their electronic and magnetic behavior, which in turn defines the functionality and stability of the system.

While there is still much effort to be made, it can already be envisaged that one could reach extreme capabilities for the

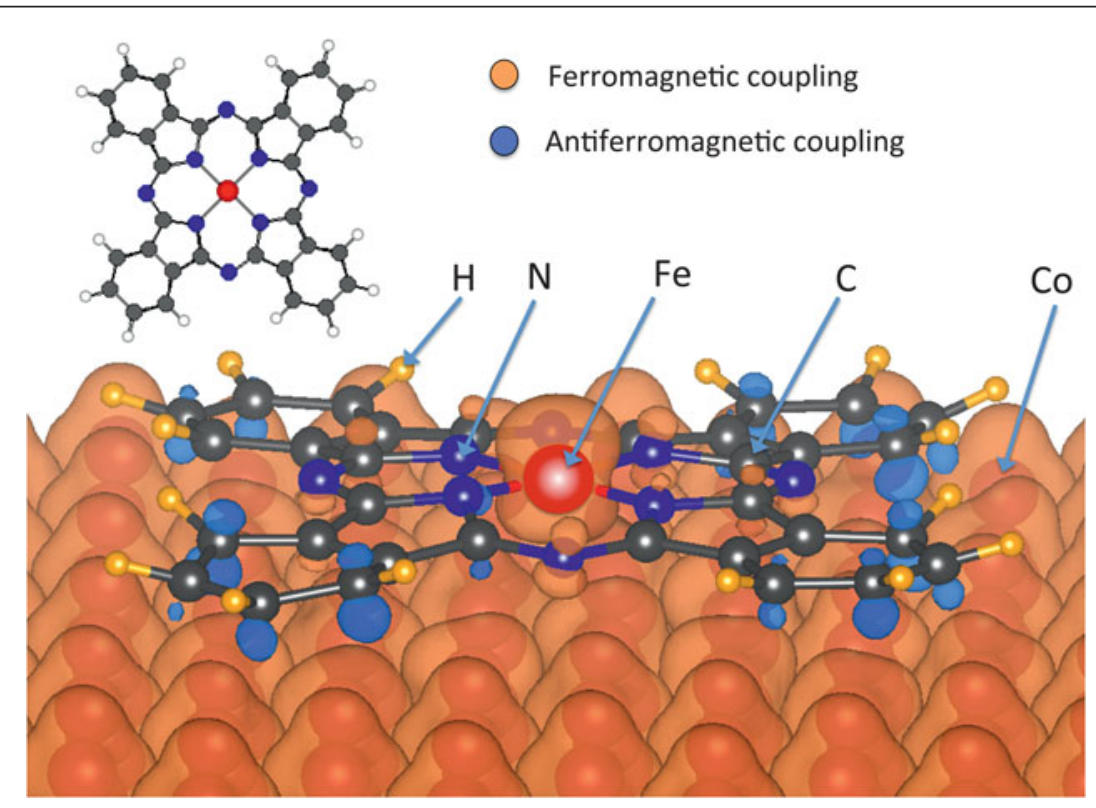

Figure 1. Illustration of the magnetic coupling arising at a metal-organic moleculesubstrate interface, leading to a spin-interface. Shown are the magnetization densities computed with density functional theory for an iron-phthalocyanine molecule adsorbed on a cobalt surface. ${ }^{3}$ The magnetization densities on the Fe and Co atoms couple ferromagnetically, as signified by the orange isosurfaces, whereas the induced magnetization density on carbon atoms couples antiferromagnetically to the Co magnetization, as shown by the light-blue isosurface (image courtesy of Kartick Tarafder). An outline of the Fe-phthalocyanine molecule is shown in the inset (blue, gray, red, and white spheres depict nitrogen, carbon, iron, and hydrogen atoms, respectively). next generation of data storage and communication devices operating at the single molecule level, reaching storage densities $>1000 \mathrm{~Tb} / \mathrm{in}^{2} .^{4-6}$ In this regard, delocalized carbonbased radical species with unpaired spin, such as phenalenyl, ${ }^{7}$ have shown promise. These phenalenyl moieties belong to the class of open-shell systems, wherein molecules are formed by the fusion of benzene rings resembling graphene fragments. The electron cloud over these rings can hybridize with the underlying surface, which defines the magnetic state of the molecule.

\section{In this issue}

The first milestone development discussed in this issue is that of the organic spin valve. From a conceptual point of view, this prototype device bears strong similarity to sensors exploiting the giant magnetoresistance (MR) effect in metallic multilayers, which has revolutionized computer hard disk technology. Spin-polarized carriers are injected from a FM electrode into an organic semiconductor and extracted by a second FM electrode. This should lead to a finite MR (i.e., a different resistance for the state with parallel and anti-parallel orientation of the magnetization vectors). However, the ability to manipulate the electron- (or hole) spin while being inside the (organic) semiconductor, and the interplay between magnetic, electric, and optical functionalities leads to a much richer application potential than just passive sensor or storage components, such as magnetic field-controlled light emission and programmable logic.

Nguyen et al. review some highlights of research on organic spin valves throughout the past decade, starting from the first report on unipolar spin valves based on small molecule organic films in $2004 .^{8}$ They also discuss targeted experiments using polymer spacer layers in which part of the hydrogen is replaced by deuterium, which has a much smaller nuclear magnetic moment. Thereby, they were able to pinpoint the significant contribution of the hyperfine field coupling to the spin relaxation of electrically injected carriers, and thus its detrimental effect on the spin diffusion length over which spin information can be transported in the organic medium. Their work triggered many successful follow-up studies. ' Yet, organic spin valves face serious challenges, both with respect to device technology — some aspects are discussed in the article - as well as their fundamental understanding. In fact, except for devices where there is direct quantum mechanical tunnel conductance, devices are typically in a regime where the well-known resistance mismatch would predict vanishing MR. Despite these difficulties, the authors report on the successful realization of a different prototype organic spintronics device: 
the spin-organic light-emitting diode. In this bipolar organic device, recombination of injected electron and hole carriers is controlled by their relative spin orientation, such that the electroluminescence emission intensity can be controlled by an external magnetic field.

While hyperfine field coupling in spin valves can be detrimental, in other approaches, the spintronic functionality fully relies on the presence of these hyperfine fields. Wohlgenannt et al. discuss an entirely complementary route to organic spintronics, namely, large room-temperature MR effects in organic devices without a FM electrode and with only the application of milliTesla magnetic fields. Rather than depending on finite spin polarization, as induced by FM electrodes in spin valve geometry, the intrinsic MR is a purely kinetic effect and is related to spin correlations between pairs of electrically injected carriers. Intriguingly, the spin dynamics of these pairs, as governed by a balance of randomly oriented hyperfine fields and globally applied external fields, is very similar to the spin dynamics of optically excited electron-hole pairs in cryptochrome proteins, nowadays thought to be relevant for the avian compass of migratory birds. The authors discuss the principles of how the spin dynamics can lead to MR in organic electronic devices, the possibility of controlling MR effects by design of the molecular structures, as well as recent advances toward devices exploiting the "fringe fields" from adjacent FM layers to induce new device functionality. Finally, they report on recently discovered giant MR effects that were found in single molecular wires, a development that could pave an alternative way toward spintronics based on a single or just a few molecule(s).

This drive toward controlling spintronics at the molecular scale is continued in the article by Atodiresei and Raman on interface-assisted spintronics. The focus is on understanding interfacial phenomenon, in particular spin-dependent chemistry and physics due to adsorption of molecules on magnetic surfaces, explored both experimentally and theoretically. It is shown that the magnetic properties of the moleculethe electronic interface spin-polarization and magnetic exchange coupling — can be tuned by appropriate choice of the organic molecules and magnetic surface. The article discusses the limitations of DFT calculations of the interfacial energies involved in organic-metal interfaces. Using ab initio DFT studies, they provide direct insights into the complex interface interactions between an organic aromatic molecule and a magnetic surface that form the hybrid interface. They show, by constructing a molecular device using phenalenyl molecules as templates, that they could engineer interfacial spin transfer resulting from $\pi$-d hybridization, giving rise to a magnetic exchange interaction with the surface of a ferromagnet (see Figure 2). As a result, the device displayed unexpected (neither predicted nor seen before) interfacial MR near room temperature, wherein the magnetic molecule showed a welldefined magnetic hysteresis on FM surfaces and magnetic hardening due to molecular chemisorption. These results herald the emergence of a new subfield of interface-assisted molecular spintronics, with an enormous scope to unravel molecular level spin functionalities. The possibility to template molecules on surfaces opens doors to build next-generation molecular memory devices with read/write capabilities as well as quantum memory and computing devices.

The article by Galbiati et al. deals with spin-dependent hybridization at FM metal/molecule interfaces. As they discuss, an induced spin polarization on molecular orbitals is observed, due to spin-dependent broadening $(<\mathrm{meV}$ for weak coupling to $\sim \mathrm{eV}$ for stronger interactions), as well as an energy shifting of the molecular levels by chemical interactions. Based on this, an effective electrode called "spinterface" is defined by considering the original FM electrode together with the first spin hybridized molecular layer at the interface. They emphasize the role of this on the interfacial spin polarization, giving two examples of enhancement and reversal of sign and how spin-hybridized states can drastically influence the spin transport properties of molecular spintronic devices.

Reaching out to magnetic interaction at the individual molecule level over a magnetic surface, Brede and Wiesendanger report the remarkable induced magnetism, individually observable in various sections of a single molecule due to chemical/ physical interactions. They applied state-of-the-art spin-polarized

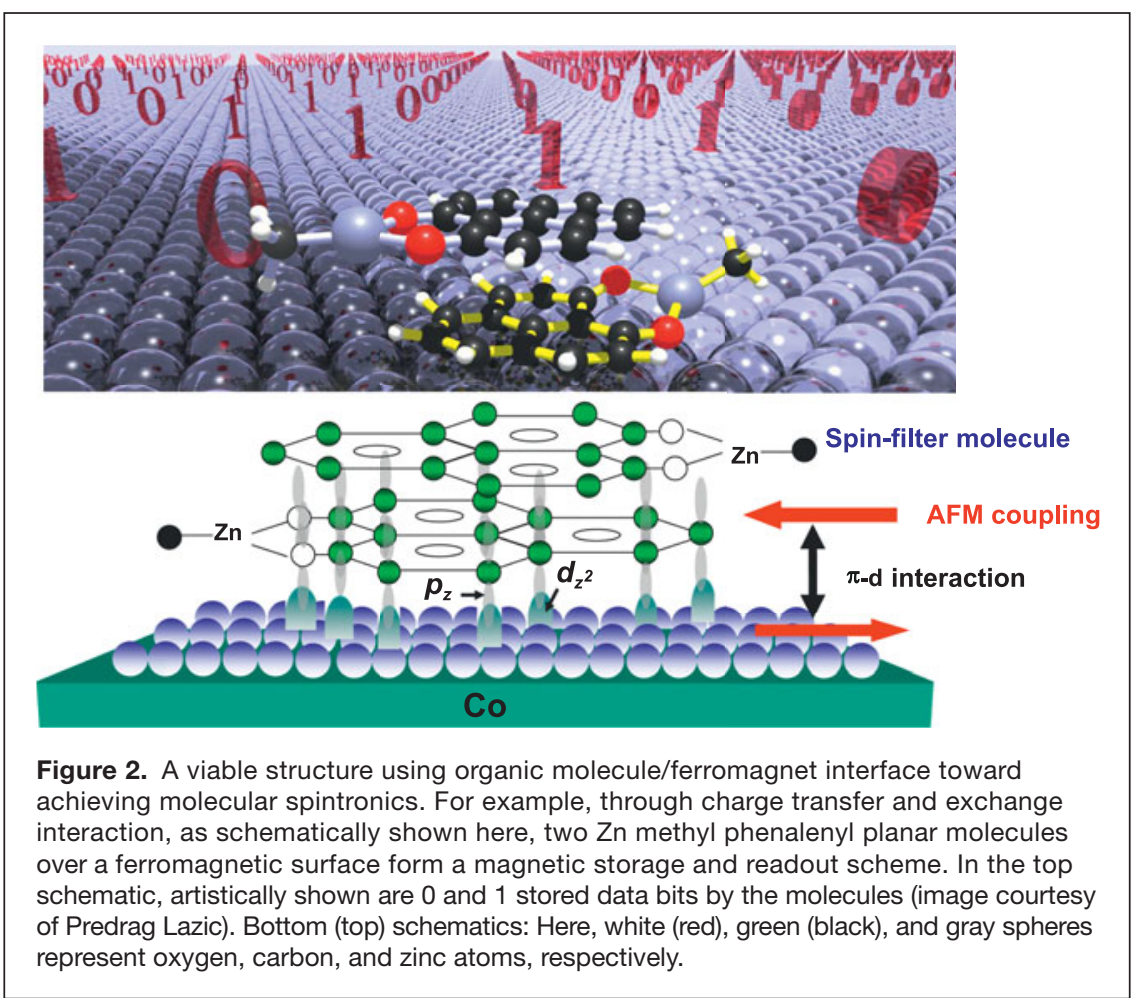


scanning tunneling microscopy and spectroscopy to show how this technique can provide unprecedented insights into organic/ magnetic interfaces with atomic-scale spatial resolution and high-energy resolution - a step toward tailoring such interfaces in order to increase spintronic device efficiency. The spin-resolved data provide not only precise experimental information on how the interaction proceeds and where the interaction takes place on the molecule to achieve, for a given energy, the desired spin-filtering effect (e.g., on the organic ligand or the central metal atom), but also the surprising observation of inversion of spin polarization on the organic ligand with respect to the FM surface. These studies yield information about the spatial distribution of the molecular spin polarization at a given energy and thus greatly assist in understanding other results using planar device structures such as organic spin valves.

The spin function of molecules reaches an additional dimension in spin-crossover (SCO) molecules, characterized by magnetic bi-stability (i.e., two different spin states on the molecule are possible). In such molecules, their magnetic spin states can be addressed and reversibly switched by applying an external stimulus such as temperature, pressure, or light irradiation. SCO molecules are accordingly considered to be promising materials for various technological applications, such as spintronics devices, ${ }^{5}$ photo-switches, ${ }^{10}$ color displays, ${ }^{11,12}$ and information storage units. ${ }^{13}$ Saha-Dasgupta and Oppeneer review the status of computational design of SCO molecules. Until recently, and in spite of the technological relevance of SCO materials, the origin of the SCO transition was not well understood. In the last few years, remarkable progress has been achieved. Ab initio electronic structure calculations can now provide an accurate quantitative description of SCO materials at low temperatures (typically $0 \mathrm{~K}$ to $50 \mathrm{~K}$ ), and by combining these with ab initio molecular dynamics simulations, the full temperature response can be simulated. Following this route, it can be shown that the temperatures at which SCO transitions occur can be rigorously predicted. These findings underscore that ab initio computational design of suitable SCO materials and their functionalities have come within reach.

\section{Conclusion}

Together, the articles in this issue of MRS Bulletin emphasize the wealth of spin functionalities that are encompassed in molecular materials. Unlocking the potential of these low-cost and easyto-produce materials for molecular spintronics applications will be a major step forward on the road to sustainable development. In this highly multidisciplinary area of research covered in this issue, we hope the reader sees a deep interconnection between the chemistry, physics, and materials aspects. The fundamental physical interactions, involving charge/spin exchange, give rise to many exciting phenomena, which offer a clear potential for device technology. It should provide an impetus for a flexible and scalable bottom-up approach toward full organic technology for a large number of applications - in sensing, molecular level magnetic memory, quantum information, or enhancing light conversion in LEDs. As there are no less fundamental implications, the recently performed research can be seen as a new milestone for its strong interdisciplinary nature, with still a vast remaining area to explore both theoretically and experimentally.

\section{Acknowledgment}

J.S.M. thanks funding support from NSF Grant DMR-1207469 and ONR Grant N00014-13-1-0301.

\section{References}

1. A. Aviram, M.A. Ratner, Chem. Phys. Lett. 29, 277 (1974).

2. A. Scheybal, T. Ramsvik, R. Bertschinger, M. Putero, F. Nolting, T.A. Jung, Chem. Phys. Lett. 411, 214 (2005).

3. S. Lach, A. Altenhof, K. Tarafder, F. Schmitt, M.E. Ali, M. Vogel, J. Sauther, P.M. Oppeneer, C. Ziegler, Adv. Funct. Mater. 22, 989 (2012).

4. M. Verdaguer, Science 272, 698 (1996).

5. T. Miyamachi, M. Gruber, V. Davesne, M. Bowen, S. Boukari, L. Joly, F. Scheurer,

G. Rogez, T.K. Yamada, P. Ohresser, E. Beaurepaire, W. Wulfhekel, Nat. Commun. 3, 938 (2012).

6. A.R. Rocha, V.M. García-Suárez, S.W. Bailey, C.J. Lambert, J. Ferrer, S. Sanvito, Nat. Mater. 4, 335 (2005).

7. R.C. Haddon, Nature 256, 394 (1975).

8. Z.H. Xiong, D. Wu, Z.V. Vardeny, J. Shi, Nature 427, 821 (2004).

9. V.A. Dediu, L.E. Hueso, I. Bergenti, C. Taliani, Nat. Mater. 8, 707 (2009)

10. S. Decurtins, P. Gütlich, C.P. Köhler, H. Spiering, A. Hauser, Chem. Phys. Lett. 105, 1 (1984)

11. S. Bonhommeau, G. Molnár, A. Galet, A. Zwick, J.A. Real, J.J. McGarvey, A. Bousseksou, Angew. Chem. Int. Ed. 44, 4069 (2005).

12. S.-i. Ohkoshi, K. Imoto, Y. Tsunobuchi, S. Takano, H. Tokoro, Nat. Chem. 3, 564 (2011).

13. 0. Kahn, C. Jay-Martinez, Science 279, 44 (1998).

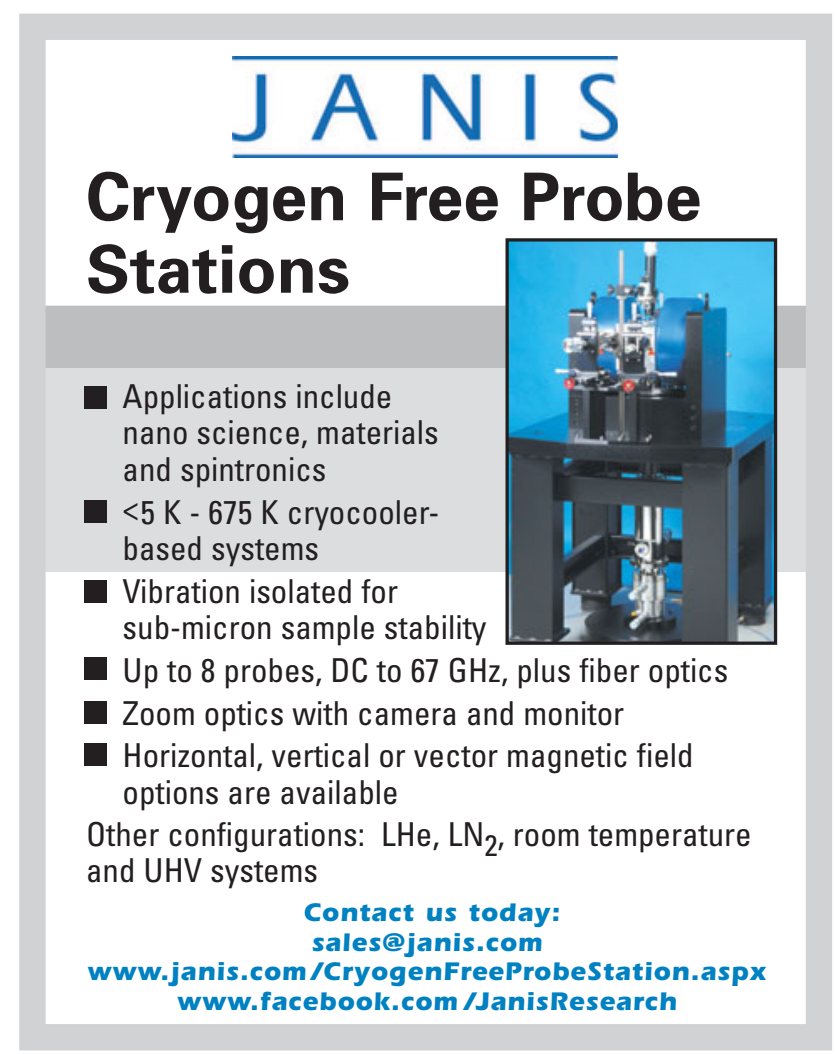

